\title{
Evaluation of a Reduced Contrast Media Protocol in Thoracoabdominal Aortic High Pitch CT-Angiography
}

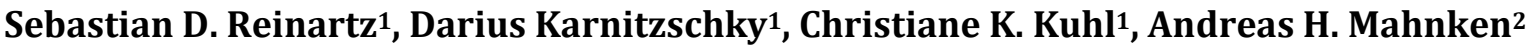 \\ ${ }^{1}$ Department of Diagnostic and Interventional Radiology, University Hospital, Aachen, Germany \\ ${ }^{2}$ Department of Diagnostic and Interventional Radiology, University Hospital, Marburg, Germany \\ Email:sreinartz@ukaachen.de
}

Received 3 October 2015; accepted 1 December 2015; published 4 December 2015

Copyright (C) 2015 by authors and Scientific Research Publishing Inc.

This work is licensed under the Creative Commons Attribution International License (CC BY). http://creativecommons.org/licenses/by/4.0/

\section{Open Access}

\begin{abstract}
Objectives: The aim is to evaluate contrast enhancement and density distribution in non-ECGsynchronized high-pitch aortoiliacal CT-angiography. Methods: 84 patients underwent clinically indicated thoracoabdominal CTA between February 2011 and May 2012 using high-pitch technique for arterial phase and an individually tailored contrast-media protocol depending on weight-class and scan duration. Standardized measurement of intravascular density is performed and accessed by multi plane reformations (MPR) orthogradly to vessels centerline. Results for 1.5 cm slices are statistically evaluated on per slice, per vessel segment ascending aortic, aortic arch, descending and abdominal aorta, right and left iliacal artery (common and external) and per patient level, regarding left-ventricular function and renal impairment. Results: In mean $53.1 \pm 7.4$ $\mathrm{ml}$ contrast-medium is administered for aortoiliacal examination. 4841 slices with mean density $280.0 \pm 63 \mathrm{HU}$ are analyzed. In 84.2\% $(n=4074)$, diagnostic quality is delivered. Regarding vessel segments, mean enhancement scored $\geq 255.5$ except weight-class 5. Adjusted to homogeneity of bolus by excluding examinations with failed bolus triggering (14\%), weight-class 1 to 3 provides $100 \%$ to $75 \%$ entirely diagnostic datasets, where amount is inadequate in weight-classes $4(62 \%)$ and 5 (2\%). Conclusions: CM protocol produces moderate but diagnostic vessel enhancement with little CM (mean $=50.7 \pm 6 \mathrm{ml}$ ) in patients smaller than $86 \mathrm{~kg}$ (weight-class 1 to 3 ) independently to left ventricular or renal function. Failure rate requires precise bolus triggering.
\end{abstract}

\section{Keywords}

Aortic Computed Tomography, Angiography, Radiologic Technology 


\section{Introduction}

Multislice spiral CT (MSCT) rapidly developed during the last decade, admitting routine use of CT angiography of aorta, peripheral vessels or coronaries. Simultaneously, contrast medium (CM) application strategies had to be adjusted for optimizing use of this technique [1]. Since coming up of high-pitch technique by dual source CT in 2005, examinations of the entire trunk in less than 5 seconds became possible, again requiring modifications in CM protocols [2] [3].

Sophisticated application of CM implicates great savings capacities on CM-volume, because vessel tree has to be contrasted only during short time of data acquisition for angiographic reasons. Consequently, individually tailored CM protocol harbors great benefit for patients, especially in case of comorbidities like impaired renal and cardiac function and in older patients. This is the case in particular in patients requiring TAVI procedure [4].

Recent strategies in CM management [3] depend on a complex relationship between patient derived factors like cardiac output, weight or underlying pathologies, CT scanner properties (e.g. scan duration) and CM-protocol dependent features such as iodine mass, injection duration or injection pattern. These factors primarily affect either magnitude or timing of enhancement or both in a proportional or inverse relationship [5]. Besides these considerations, only some studies practically apply an individually tailored CM protocol [6] [7]. Additionally, as ECG-synchronized high-pitch examinations are primarily intended for comprehensive coronary CTA [7]-[9], non-ECG-synchronized aortic CTA is less explored [4] [10] [11]. Consequently, purpose of our study is to retrospectively evaluate performance of a weight dependent [5], individually tailored contrast protocol in non-ECG-synchronized aortic CTA using high pitch technique, which practically implements relationship between patients, CM and hardware factors. Therefore, we hypothesize that in these ultrashort examinations scan durations alone are not useful for defining total injection duration even with excellent timing, i.e. a fixed security time interval of 8 seconds is added to ensure a so called physiologic minimum contrast enhancement. For compensational reasons, bolus shaping, using a biphasic CM protocol, is developed to reduce total amount of CM. In opposition to other studies, evaluation of intravascular enhancement and density distribution is performed, and additionally focus is set on effects of impaired cardiac or renal function.

\section{Material and Methods}

\subsection{Patients}

Between February 2011 and May 2012 we retrospectively enrolled 85 consecutive patients with clinically indicated not ECG synchronized high-pitch DSCT of the aorta and iliacal vessels. 1 patient had to be excluded due to incomplete physiological data and corrupt protocol file. The resulting 32 (38\%) women and 52 (62\%) men had a mean $(\mathrm{SD})$ age of $66.69( \pm 13.8)$ years.

\subsection{Preparations, Image Protocol and Dose Estimation}

Patient's weight and height as well as informed consent were routinely obtained as standard preparation before examination. Administration of drugs was nor intended or given. The complete cohort underwent mono-, bi- or triphasic CTA where only arterial phase was examined using high-pitch technique. It is optionally framed by non-ECG synchronized helical examinations with conventional pitch, recording native and/or venous phase. Indications were suspected pathology like aneurysm or dissection $(n=28,33 \%)$, as a post-interventional or postsurgical control ( $n=25,30 \%)$ or for follow-up purposes $(n=21,25 \%)$ and for other reasons $(n=10,8 \%)$. The scanning range extended from the thoracic inlet to the ischial tuberosities, in 14 cases only thoracic aorta was examined.

Examinations were provided using a first generation DSCT (Somatom Definition, Siemens Healthcare Sector, Forchheim, Germany) with two perpendicularly mounted tube-detector systems (32 slices) using flying z-spot technology. Thus, only limited Field of View (FoV) was provided in case of high-pitch usage. DSCT standardly used a collimation of $2 \times 32 \times 0.6 \mathrm{~mm}$, a gantry rotation time $330 \mathrm{~ms}$ and a tube voltage of $120 \mathrm{kVp}$. The tube current-time product yielded $400 \mathrm{mAs}_{\text {rot }}$.

Pitch varied during study time due to department modifications in high-pitch examinations, a constant value of 1.2 was used in any conventional setting.

Mean estimated radiation dose was calculated by multiplying the dose length product (DLP) and the conversion coefficient of $0.014 \mathrm{mSv} \cdot \mathrm{mGy}^{-1} \cdot \mathrm{cm}^{-1}$ for thoracic or $0.015 \mathrm{mSv} \cdot \mathrm{mGy}^{-1} \cdot \mathrm{cm}^{-1}$ thoracoabdomnial examinations. 


\subsection{Ethics Commission}

Approval of the local ethics commission was successfully obtained.

\subsection{Contrast Media}

It is known, that patients factors (weight and cardiac output), CT scanning factors (i.e. scan duration) and CM factors relevantly influence contrast enhancement in the aorta [2] [3] [5].

The latter largely depends on the delivered Iodine flux or mass, defined by Iodine concentration, injection duration and delivery rate. This factor cannot exceed 8 - $10 \mathrm{ml} / \mathrm{s}$ without highly increasing risk for extravasation. Beside this, patient's convenience suffers during administration, too, if an appropriate injection side is available. But, injection duration also influences peak vessel enhancement and bolus length. Therefore, a monophasic protocol would require inadequate high amounts of CM for enhancement of complete vessel tree, endangering renal function. Therefore, shaping the bolus by introducing a biphasic CM protocol is an appropriate technique to economize CM-volume. Core idea of our protocol is to link patient, scanning and CM factors practically to provide minimal CM usage and diagnostic enhancement of the vessels during arterial phase.

For this reason, we stratified according to Fleischmann et al. [5] patient's weight practically into five classes (less than $56 \mathrm{~kg}, 56$ - $65 \mathrm{~kg}, 66$ - $85 \mathrm{~kg}, 86$ - $95 \mathrm{~kg}$ and more than $95 \mathrm{~kg}$ ) and connected weight-class with increasing Iodine delivery rate from 1.5 up to $1.9 \mathrm{~g}$ Iod/s (Table 1). By limiting the first protocol phase (Phase 1)

Table 1. CM Protocol: TID means total injection duration. It is the addition of scan duration plus Security Time Interval (STI), which is set to 8 seconds. Iopromide $0.37 \mathrm{~g} \mathrm{Iod} / \mathrm{ml}$ was used as contrast media. Therefore, CM rate was calculated by multiplying Iodine flux with Iopromide Iodine concentration for each weight-class. By defining duration in Phases 1 - 3, volume is computed by multiplying rate with duration. In Phases 4 and 5, volume is defined and rate is transferred from previous phases. Therefore duration is calculated by dividing volume and rate.

\begin{tabular}{|c|c|c|c|c|c|c|}
\hline Phase No. & $\begin{array}{l}\text { Weight } \\
\text { (kg) }\end{array}$ & $\begin{array}{l}\text { Iod-flux } \\
\text { (g Iod/s) }\end{array}$ & $\begin{array}{l}\text { CM Rate } \\
(\mathrm{ml} / \mathrm{s})\end{array}$ & $\begin{array}{l}\text { Duration } \\
\text { (s) }\end{array}$ & $\begin{array}{l}\text { CM Volume } \\
\text { (ml) }\end{array}$ & $\begin{array}{l}\text { Saline Volume } \\
\text { (ml) }\end{array}$ \\
\hline \multirow{5}{*}{$\begin{array}{c}\text { Phase 1: } \\
\text { CM } \\
\text { fast rate }\end{array}$} & $<56$ & 1.5 & fast-rate & 5 & \multirow{5}{*}{$\begin{array}{l}\text { Variable, weight-class } \\
\text { dependent calculated } \\
\text { by formula: } \\
\text { Volume = rate } \\
\text { duration }\end{array}$} & \multirow{15}{*}{$\begin{array}{c}\text { Variable, weight-class } \\
\text { dependent calculated } \\
\text { by formula: } \\
\text { Volume = rate } \\
\text { duration }\end{array}$} \\
\hline & $56-65$ & 1.6 & fast-rate & 5 & & \\
\hline & $66-85$ & 1.7 & fast-rate & 5 & & \\
\hline & $86-95$ & 1.8 & fast-rate & 5 & & \\
\hline & $>95$ & 1.9 & fast-rate & 5 & & \\
\hline \multirow{5}{*}{$\begin{array}{c}\text { Phase 2: } \\
\text { CM } \\
\text { slow rate }\end{array}$} & $<56$ & 1.2 & slow-rate & TID-Duration (Phase 1) & \multirow{5}{*}{$\begin{array}{l}\text { Variable, weight-class } \\
\text { dependent calculated } \\
\text { by formula: } \\
\text { Volume = rate } \\
\text { duration }\end{array}$} & \\
\hline & $56-65$ & 1.3 & slow-rate & TID-Duration (Phase 1) $_{\text {(1) }}$ & & \\
\hline & $66-85$ & 1.4 & slow-rate & TID-Duration ${ }_{\text {(Phase 1) }}$ & & \\
\hline & $86-95$ & 1.5 & slow-rate & TID-Duration (Phase 1) & & \\
\hline & $>95$ & 1.6 & slow-rate & TID-Duration (Phase 1) & & \\
\hline \multirow{5}{*}{$\begin{array}{c}\text { Phase } 3 \text { : } \\
\text { saline chaser }\end{array}$} & $<56$ & & slow-rate & 10 & & \\
\hline & $56-65$ & & slow-rate & 10 & & \\
\hline & $66-85$ & & slow-rate & 10 & & \\
\hline & $86-95$ & & slow-rate & 10 & & \\
\hline & $>95$ & & slow-rate & 10 & & \\
\hline \multirow{5}{*}{$\begin{array}{l}\text { Phase 4: } \\
\text { CM }\end{array}$} & $<56$ & variable & slow-rate & \multirow{5}{*}{$\begin{array}{l}\text { Variable, weight-class } \\
\text { dependent calculated by } \\
\text { formula: } \\
\text { duration = volume/rate }\end{array}$} & $120-\mathrm{CM}_{\text {(Phase 1+2) }}$ & \\
\hline & $56-65$ & variable & slow-rate & & $120-\mathrm{CM}_{\text {(Phase 1+2) }}$ & \\
\hline & $66-85$ & variable & slow-rate & & $120-\mathrm{CM}_{\text {(Phase 1+2) }}$ & \\
\hline & $86-95$ & variable & slow-rate & & $120-\mathrm{CM}_{\text {(Phase 1+2) }}$ & \\
\hline & $>95$ & variable & slow-rate & & $120-\mathrm{CM}_{(\text {Phase 1+2) }}$ & \\
\hline \multirow{5}{*}{$\begin{array}{c}\text { Phase } 5 \text { : } \\
\text { saline chaser }\end{array}$} & $<56$ & & slow-rate & & & 30 \\
\hline & $56-65$ & & slow-rate & \multirow{4}{*}{$\begin{array}{l}\text { variable, weight-class } \\
\text { dependent calculated by } \\
\text { formula: } \\
\text { duration = volume/rate }\end{array}$} & & 35 \\
\hline & $66-85$ & & slow-rate & & & 40 \\
\hline & $86-95$ & & slow-rate & & & 45 \\
\hline & $>95$ & & slow-rate & & & 50 \\
\hline
\end{tabular}


to 5 seconds, the fast rate injection is build. In order to reduce total CM volume, a second injection phase with slow rate (Phase 2) was defined by connecting scan duration (SCD) and fixed security time interval (STI = 8 seconds) with total injection duration (TID [5]) by the following equations:

$$
\begin{aligned}
\text { TID } & =\text { SCD }+ \text { STI } \\
\text { Duration }_{(\text {Phase 2) }} & =\text { TID }- \text { Duration }_{(\text {Phase 1) }}
\end{aligned}
$$

The fixed security time interval was defined to preserve minimum CM amount in case of short acquisition times, especially in high-pitch examtinations, and was empirically set to 8 seconds. Again, weight-class dependent crescent Iodine-flux was defined (1.2 to $1.6 \mathrm{~g} \mathrm{Iod} / \mathrm{s}$ ), building the second phase of the biphasic CM protocol for bolus shaping purposes. The following saline chaser lasts fixed 10 seconds and uses previously defined slow delivery rate.

By clinical necessity of imaging aorta in venous phase, this biphasic CM protocol with saline chaser was enlarged by a monophasic CM protocol (Phase 4) with an additional saline chaser (Phase 5). Because department's protocol recommends a fixed total volume of $120 \mathrm{ml} \mathrm{CM}$, the complementary amount of CM (120 minus CM, delivered in Phase 1 and 2) was applied in Phase 4, using previously applied slow delivery rate. The weight-class dependent saline chaser $(30-50 \mathrm{ml} \mathrm{NaCl}$ ) was delivered at the same rate in Phase 5. CM volume range amounted therefore $41-74 \mathrm{ml}$ for arterial enhancement, whereas total volume was fixed to $120 \mathrm{ml}$ to perform an examination in venous phase due to department's standard operation procedures. To avoid calculation errors, a self-made dedicated software (CM-Sherpa), programmed with Delphi 6 (Borland, Austin, Tx, USA) was used. In this tri- or pentaphasic protocol Iopromide (Ultravist $370 \mathrm{mg}$, Schering, Berlin, Germany) was applied.

To contribute to differing patients cardiac output, bolus-tracking technique was applied, commencing data acquisition after a threshold of $180 \mathrm{HU}$ was reached. The ROI was positioned in the descending aorta above the diaphragm.

\subsection{Image Postprocessing and Measuring}

To provide accurate measurement of vascular enhancement by standardized slice thickness, isotropic reconstructions (1 mm slices) were transferred to the MMWP-workstation (Leonardo Multimodality Workplace MMWP, Siemens). By using dedicated software (InSpace, Siemens), a vessel centerline (Figure 1(a)) regarding complete aortic and right common and external iliacal arterie was manually defined. Then multi plane reconstructions orthogradely to centreline were calculated with $1.5 \mathrm{~cm}$ slice thickness and $1.5 \mathrm{~cm}$ increment (Figure 1(b) and Figure 1(c)). The corresponding procedure was performed for left iliacal vessels (common and external iliacal artery). A standardized region of interest (ROI) of $0.82 \mathrm{sqcm}$ was placed in aorta for measuring density. Additionally, slice's localisation was documented with distance to coronary branches and by noting down aortic branches (brachiocephalic trunc, left carotid artery, left subclavian artery, coeliac trunk, superior mesenteric artery, both renal arteries) as landmarks. First coronary branch downstream was defined as starting point instead of aortic root because of practical reasons to avoid collisions with aortic valve calcifications. Iliacal arteries required a standardized ROI of $0.22 \mathrm{sqcm}$. In case of covering pathology (dissection membrane, exophytic calcifications etc) or foreign bodies (i.e. Stents etc.) with ROI, it was manually moved or slightly reduced to avoid collision. By impossibility to maintain an adequate area of $0.2 \mathrm{sqcm}$ for aorta, $0.1 \mathrm{sqcm}$ for iliacal vessels, the slice was excluded from evaluation.

\subsection{Visual Assessment}

Radiologists with three years (R, SD) experience in CTA and a doctoral candidate (K, DJ) evaluated in consensus appropriateness of reconstructions. In case of problems, a radiologist with eleven years experience (M, AH) was consulted. Data harvesting was performed using a MS-Access Database (Microsoft Corporation, Redmond, WA, USA).

\subsection{Statistical Analysis}

Using SPSS 20 (IBM Corporation, NY, USA) data was statistically analysed. Continuous variables are expressed as mean \pm standard deviation (SD), categorical variables as numbers and percentages. To extract statistical significance in continuous data, T-test was performed for paired data to compare vessel segments in one 


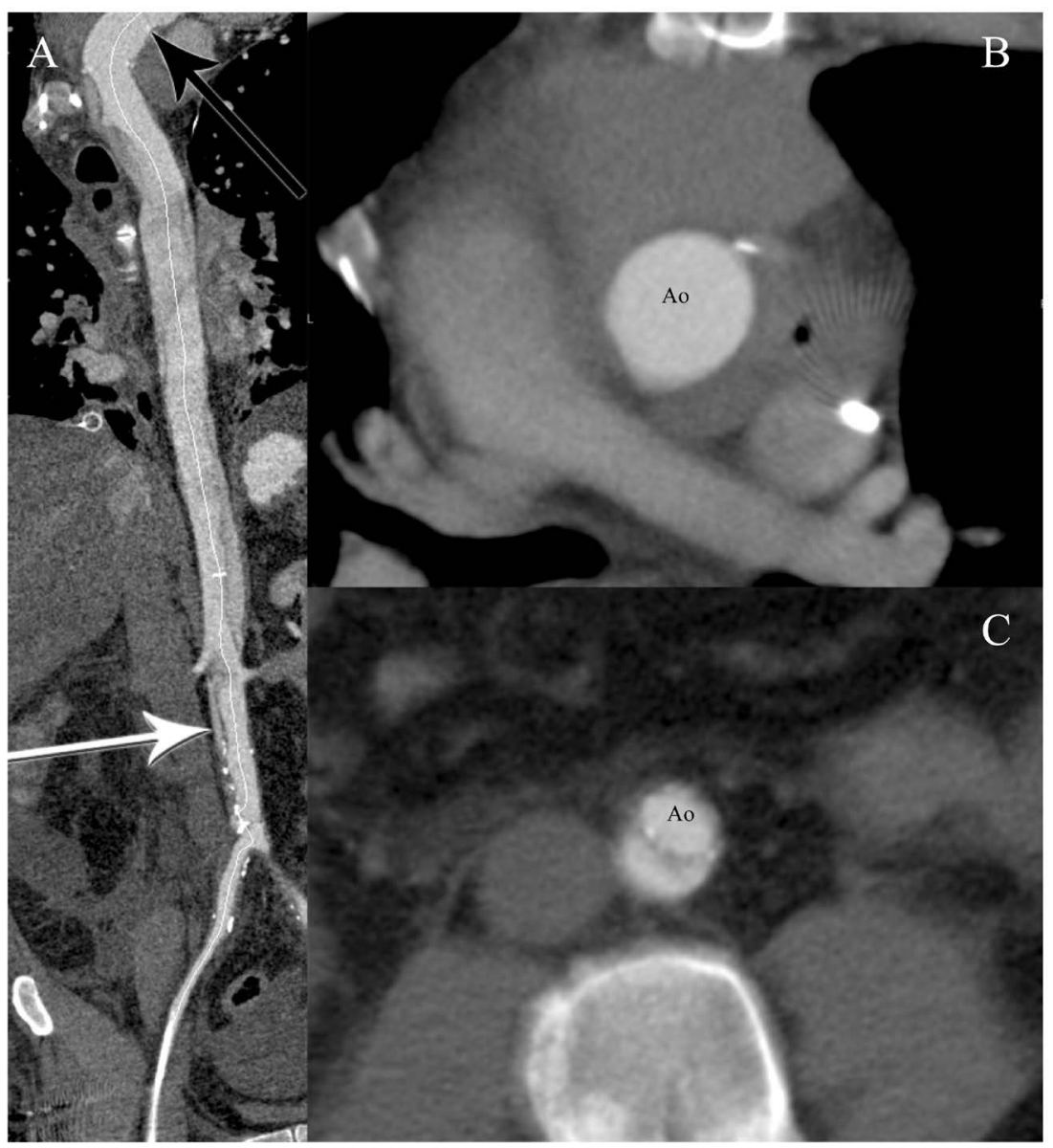

Figure 1. (A): A vessel centerline in the aorta and right common and external iliacal arterie was manually defined. In this case a 74 year old woman with Stanford A dissection was evaluated. Then multi plane reformations orthogradely to the centerline were calculated exemplarily at the height of the coronaries ((B) black arrow) the abdominal aorta ((C) white arrow). Then intravascular density was measured with standardized region of interest. Ao $=$ Aorta.

patient or unpaired data to prove differences in means by slice or patient. A $P$-value below 0.05 was assumed to be statistically significant. In categorical data $\chi^{2}$-test was applied for comparison of frequencies in patients' characteristics.

Comparison of vessel enhancement was performed on per slice, per vessel segment and per patient level. In per segment analysis, vessel tree was subdivided into thoracic ascending and descending aorta, aortic arch, abdominal aorta and iliacal arteries (combination of external and common branch) distinguishing between left and right side.

In order to measure homogeneity of contrast medium bolus and to register failure in bolus triggering, normal distribution of density was assumed and a twofold standard deviation equal to or less than $75 \mathrm{HU}$ was assumed to describe homogeneity of bolus. That means $95.4 \%$ of measures regarding the relevant patient are within an interval of $150 \mathrm{HU}$.

Due to observational study design a cohort of 84 consecutive patients was assumed being representative.

\section{Results}

\subsection{Cohort Analysis}

Our cohort with 84 patients showed no gender specific statistically significant differences besides weight and height in neither coronary risk factors (Table 2) like arterial hypertension, obesity etc. nor in prevalence of 
Table 2. Patients demographics and risk factors respectively organ dysfunction is noted regarding gender and weight-class. Normal distribution of patients regarding weight-class was proven.

\begin{tabular}{|c|c|c|c|c|c|c|c|c|}
\hline & \multirow[b]{2}{*}{$\begin{array}{l}\text { Entirecohort } \\
\quad(n=84)\end{array}$} & \multirow[b]{2}{*}{$\begin{array}{c}\text { Male } \\
n=52(62 \%)\end{array}$} & \multirow[b]{2}{*}{$\begin{array}{c}\text { Female } \\
n=32(38 \%)\end{array}$} & \multicolumn{5}{|c|}{ Weight-class } \\
\hline & & & & $\begin{array}{c}1 \\
n=4\end{array}$ & $\begin{array}{c}2 \\
n=21\end{array}$ & $\begin{array}{c}3 \\
n=33\end{array}$ & $\begin{array}{c}4 \\
n=14\end{array}$ & $\begin{array}{c}5 \\
n=12\end{array}$ \\
\hline \multicolumn{9}{|l|}{ Demographics } \\
\hline Age (years) & $66.69 \pm 13$ & $66.1 \pm 11$ & $67.6 \pm 16$ & $65.5 \pm 15$ & $64.3 \pm 16$ & $69.2 \pm 12$ & $71.1 \pm 11$ & $59.0 \pm 13$ \\
\hline Death & $4(5 \%)$ & $3(75 \%)$ & $1(25 \%)$ & $1(1.2 \%)$ & 0 & $2(2.4 \%)$ & $1(1.2 \%)$ & 0 \\
\hline Weight (kg) & $78.5 \pm 17.4$ & $83.8 \pm 16.4$ & $69.8 \pm 15.4$ & $51.3 \pm 3$ & $61.1 \pm 3$ & $77.2 \pm 5$ & $90.1 \pm 2$ & $108.1 \pm 13$ \\
\hline Height (cm) & $171.6 \pm 9.9$ & $175.9 \pm 8.6$ & $164.43 \pm 7.4$ & $161 \pm 15$ & $164 \pm 7$ & $172 \pm 6$ & $177 \pm 11$ & $181 \pm 9$ \\
\hline \multicolumn{9}{|l|}{ Riskfactors } \\
\hline Arterial hypertension & $55(66 \%)$ & 35 (61\%) & 22 (38\%) & 3 & 14 & 23 & 10 & 7 \\
\hline Diabetes & $10(12 \%)$ & $3(30 \%)$ & 7 (70\%) & 0 & 1 & 5 & 3 & 1 \\
\hline Hypercholesterolemia & $20(24 \%)$ & $10(50 \%)$ & $10(50 \%)$ & 1 & 4 & 10 & 4 & 1 \\
\hline Obesity & $19(23 \%)$ & $10(53 \%)$ & $9(47 \%)$ & 0 & 2 & 5 & 5 & 8 \\
\hline Smoking & 19 (23\%) & $14(74 \%)$ & $5(26 \%)$ & 2 & 3 & 8 & 3 & 3 \\
\hline \multicolumn{9}{|l|}{ Left ventricular function } \\
\hline Normal & 46 (55\%) & & & & & & & \\
\hline Mildly impaired & $12(14 \%)$ & $4(5 \%)$ & $8(10 \%)$ & $1(1.2 \%)$ & $5(6 \%)$ & $3(4 \%)$ & $2(2 \%)$ & $1(1.2 \%)$ \\
\hline $\begin{array}{l}\text { Moderate tosevere } \\
\text { impaired }\end{array}$ & $8(10 \%)$ & $6(7 \%)$ & $2(2 \%)$ & 0 & $1(1.2 \%)$ & $5(6 \%)$ & $1(1.2 \%)$ & $1(1.2 \%)$ \\
\hline \multicolumn{9}{|l|}{ Renal function } \\
\hline Normal & $54(64 \%)$ & $32(38 \%)$ & $22(26 \%)$ & $2(2.4 \%)$ & 15 (18\%) & $20(24 \%)$ & $9(11 \%)$ & $8(10 \%)$ \\
\hline Impaired and dialysis & $26(31 \%)$ & $17(20 \%)$ & $9(10 \%)$ & $1(1.2 \%)$ & $6(7 \%)$ & $12(14 \%)$ & $4(5 \%)$ & $3(4 \%)$ \\
\hline
\end{tabular}

aneurysms, dissections or stent treatment. 3 (4\%) patients died during hospital stay due to underlying illness (n.s.). One adverse event occurred (1.2\%) due to application of contrast media (extravasation).

Regarding organ dysfunction 54 patients (64\%) had normal renal function, 26 patients (31\%) were noticeable with impaired glomerular filtration rate (GFR) $\leq 60 \mathrm{ml} / \mathrm{min} / 1.72 \mathrm{sqm}$ ), one patient (1.2\%) was treated with dialysis. In four cases (5\%), no renal data was available in electronic patient records.

In a subgroup of 46 patients (55\%) normal ejection fraction of the left ventricle (LV) was documented, whereas in $21 \%$ of the cases $(n=18) \mathrm{LV}$-function was not evaluated. 19 records $(22.8 \%)$ showed mild to moderate impaired LV-Function, one patient (1.2\%) suffered from heart failure due to severely impaired heart function.

\subsection{Examination Analysis and Dose Estimation}

Regarding the complete cohort, pitch ranged in-between 1.55 to 3.2 with in mean ( \pm SD) $2.67 \pm 0.6$. A mean scan duration of $4.1 \pm 1.5$ seconds caused a mean effective dose of $10.1 \pm 3.0 \mathrm{mSv}$.

In 14 examinations, only thoracic aorta was examined with a mean examined aortic length of $40.9 \pm 4.3 \mathrm{~cm}$ (range 34.5 - 49.5), an estimated effective dose $\mathrm{E}=5.2 \pm 0.73 \mathrm{mSv}(n=13)$ and with a pitch $( \pm \mathrm{SD}) 2.9 \pm 0.4$ (range 2.0 - 3.2). Scan lasted $2.1 \pm 0.32$ seconds. Three of these had mildly impaired LV-function, none of them suffered from impaired renal function (GFR $>60 \mathrm{ml} / \mathrm{min} / 1.73 \mathrm{sqm}$ ).

The residual 70 examinations were scanned in $4.5 \pm 1.3$ seconds, mean evaluated length of aorta measured $73.1 \pm 6.5 \mathrm{~cm}$ (range: 58.5 - $93 \mathrm{~cm}$ ), conditioning a mean effective dose of $11.0 \pm 2.5 \mathrm{mSv}$. Pitch $( \pm \mathrm{SD})$ scored $2.6 \pm 0.6$, ranging from 1.55 to 3.2. 17 (24\%) of the corresponding patients had impaired LV-function. In 26 (37\%) cases a reduced GFR of below $60 \mathrm{ml} / \mathrm{min} / 1.73 s q m$ was documented.

\subsection{Contrast Media Analysis}

In mean $53.1 \pm 7.4 \mathrm{ml}( \pm \mathrm{SD})$ contrast media was injected for enhancement in arterial phase, divided in Phase 1 
with $23.4 \pm 1.5 \mathrm{ml}$ and Phase 2 with $29.7 \pm 6.7 \mathrm{ml}$. Range is defined by a minimum of $41 \mathrm{ml}$ and a maximum of $74 \mathrm{ml}$. Phase 1 required a rate of $4.6 \pm 0.3 \mathrm{ml} / \mathrm{s}$, Phase $23.8 \pm 0.3 \mathrm{ml}$, while total injection duration was $12.8 \pm$ $1.6 \mathrm{ml}$. Significant less contrast media $(P=0.002)$ was administered during examinations limited to thorax $(48.1 \pm$ $5.3 \mathrm{ml}$ vs. $54.1 \pm 7.3 \mathrm{ml}$ ) and slightly but significantly shortened total injection duration was monitored (11.6 \pm 1.1 vs. $12.8 \pm 1.6$ seconds). But, there was no remarkable difference regarding CM volume in Phase 1 .

\subsection{Per Slice Analysis}

A total amount of 4841 slices was measured. 29 (0.5\%) slices had to be excluded from evaluation due to dissection ( $n=10,35 \%)$, narrowness of vessel and corruptness of centerline (each $n=6,21 \%$ ), Stent or massive calcifications (each $n=3,10 \%$ ) or aneurysm $(n=1,3 \%)$. For details see also Figure 2.

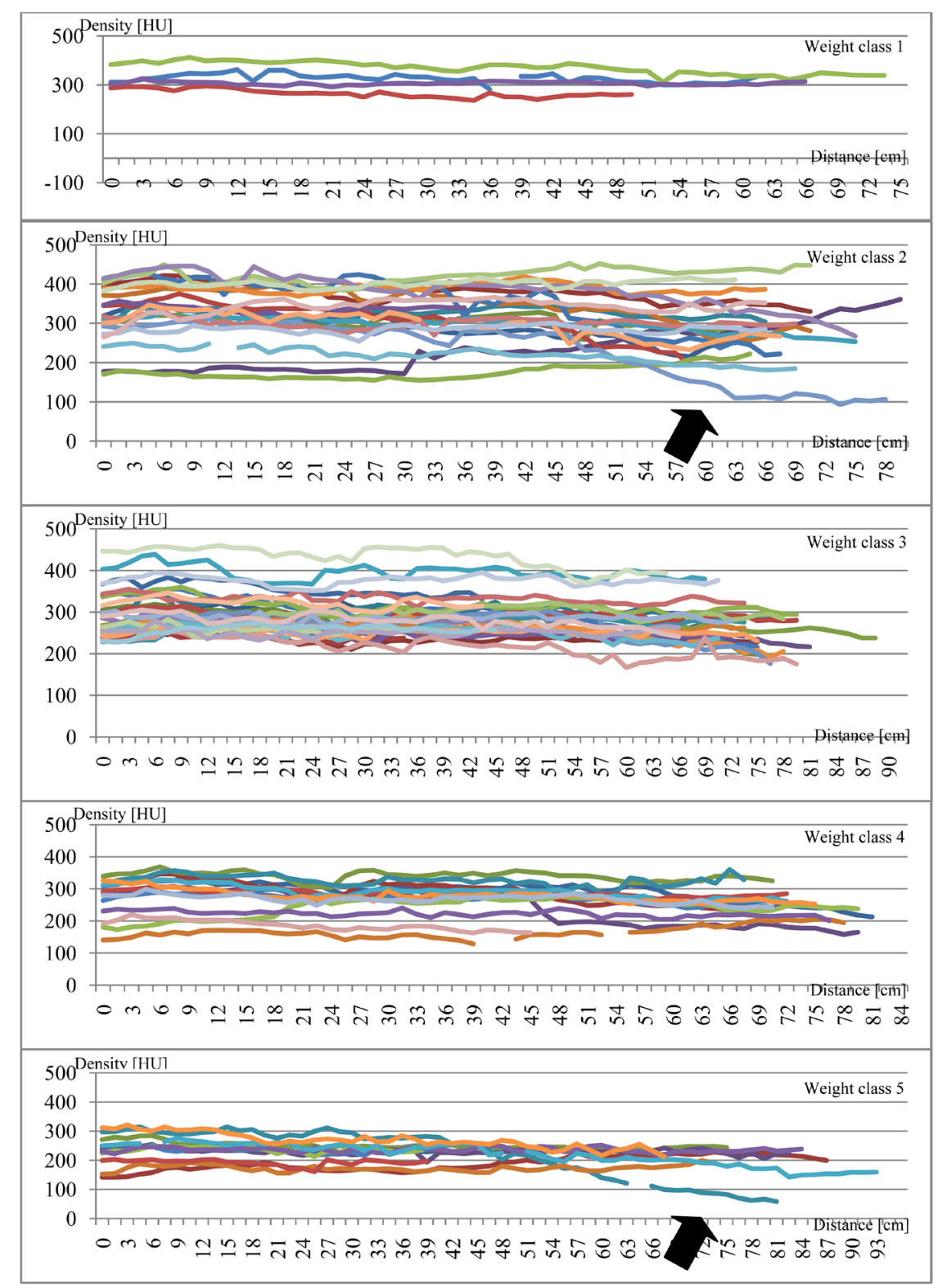

Figure 2. Each ordinate is showing density in HU, distance to coronaries in centimeters is plotted on the x-axis. Density distribution for weight-classes $1-5$ is shown in the five graphs. Two inhomogeneous bolus are marked by black arrows, indicating failure in bolus triggering. 
454 slices (9.4\%) yield a density below $200 \mathrm{HU}, 284$ (5.9\%) score in between $200 \mathrm{HU}$ and $225 \mathrm{HU}$ and 4074 (84.2\%) yield diagnostic density of greater or equal to $225 \mathrm{HU} .746$ (15.4\%) were higher than $224 \mathrm{HU}$ but lower than $250 \mathrm{HU} .3328$ (68.8\%) yield greater or equal to $250 \mathrm{HU}$, where 1721 (35.6\%) lay below $300 \mathrm{HU}$.

Mean density undercuts $225 \mathrm{HU}$ only in patients bigger than $95 \mathrm{~kg}$ (Table 3), whereas any other subgroup and weight-class yields above $(P<0.001)$.

Subgroup analysis distinguishing between normal $(n=2554$ slices) and impaired LV-function $(n=1177$ slices) reveals no significant difference ( $280.1 \pm 67$ vs. $278.9 \pm 66 \mathrm{HU})$. However, analysis of mean enhancement in patients with mildly reduced $(n=665,297.3 \pm 58 \mathrm{HU})$ vs. normal or higher reduced ejection fraction discovered statistically significant difference $(P<0.001)$.

Regarding renal function in 80 patients with documented GFR, vessels mean density scores significantly higher in patients with impaired renal function ( $296.2 \pm 65 \mathrm{HU}, n=1663$ vs. $271.3 \pm 62, n=2896), P<0.001$.

\subsection{Per Vessel Segment Analysis}

Mean vessel density scores on a per segment level at least $255.5 \mathrm{HU}$ and is therefore above $225 \mathrm{HU}$. Exception is the entire weight-class 5, ranging in mean from 201.9 to $241.4 \mathrm{HU}$. Correspondingly to per slice analysis, mean density was statistically significant $(P<0.001)$ lower for any vessel segment compared to any other weight-class.

Comparing adjacent vessel segments, average density undulates and therefore slightly rises between ascending aorta and aortic arch (n.s.) respectively descending and abdominal aorta $(P \leq 0.026)$. Density significantly declines between aortic arch and descending aorta $(P=0.002)$ respectively abdominal aorta and iliacal vessels $(P<0.001)$. This phenomenon is observable in the entire cohort and subgroups with normal ejection fraction, renal function and bodyweight 66 - $85 \mathrm{~kg}$ (class 3). A consequent decrease of mean density downstream from proximal to distal was stated only in subgroups with impaired renal function and moderate to severe LV-function.

In patients with mildy as well as moderate to severe impaired LV Function mean density falls dramatically in both iliacal arteries compared to abdominal aorta (15.8\% respectively $10.2 \%$ as well as in weight-class 5 (8.5\%) compared to mean percental decrease (5.0\%).

The number of diagnostic segments is reduced in e.g. iliacal arteries in comparison to ascending aorta. The percentage of non-diagnostic vessel enhancement rises from $10 \%$ in areas close to the heart to approx. $20 \%$ in the periphery of the aorta.

\subsection{Per Patient Analyis}

Regarding the entire collective, 49 (58\%) datasets yielded entirely greater or equal to $225 \mathrm{HU}$ for every corresponding slice. Subgroup analysis discovers $100 \%$ diagnostic datasets $(n=4$ patients) for weight-class 1 and moderately $73 \%$ in weight-class 3, which consists of 33 patients. Weight-class 2 and 4 performed $57 \%$ entirely diagnostic datasets (total amount: $n=21$ vs. 14 patients), whereas there is only one patient in subgroup 5 (8\% out of 12 patients in weight-class 5). Renal subgroups scored similar with $56 \%$ and $62 \%$ for normal $(n=54)$ and impaired GFR ( $n=26)$ as well as patients with normal, mildly reduced or unknown EF (61\%, $n=46 ; 58.3 \%, n$ $=12 ; 61 \%, n=18$ ). In subgroup with moderate to severe impaired $\mathrm{EF}$, diagnostic datasets were accessible in only three patients $(43 \%)$.

$86 \%(n=72)$ of all examinations had a twofold SD $\leq 75 \mathrm{HU}$, i.e. bolus corridor measured $150 \mathrm{HU}$, indicating homogeneity. Regarding these examinations (Table 4), 8 patients (38\%) had to be excluded in weight-class 2, each one in class $3(3 \%)$ and $4(7 \%)$ respectively 2 patients in weight-class $5(16 \%)$. Therefore, number of diagnostic examinations rises to $77 \%(n=10$ out of 13$)$ and $62 \%(n=8$ out of 13$)$ in weight-class 2 respectively 4 . In contrary, amount stagnates in class 1,3 and 5. Corresponding analysis for subgroups reveals risen but poor number of diagnostic examinations (50\% - 68\%). Number of diagnostic slices does not show relevant changes in entire cohort or subgroups on basis of homogeneity, except in patients with moderate to severe impaired LVfunction $(72 \%$ to $82 \%)$.

\section{Discussion}

\subsection{Assessment of Demographics}

This study evaluated aortic and iliacal contrast enhancement and density distribution using a weight-class tailored 


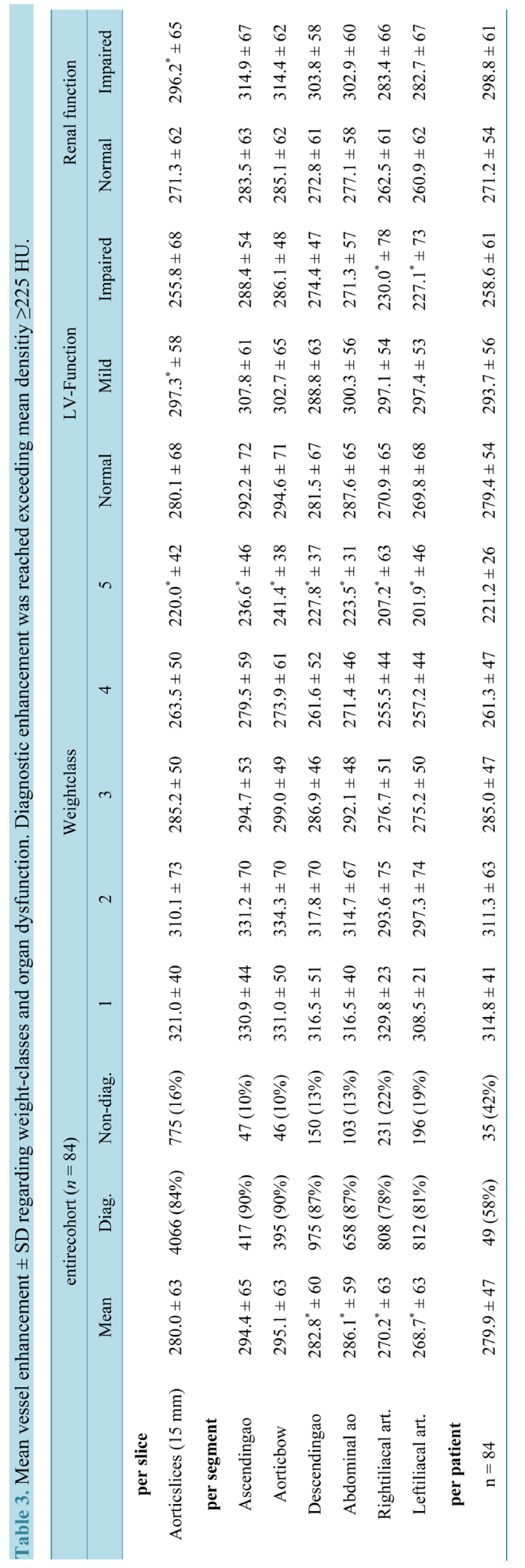


Table 4. Homogeneity: Diagnostic slices or examinations, exceeding mean density $\geq 225 \mathrm{HU}$ in each slice, are noted with percentage regarding subgroup and adjusted to homogeneity of bolus. $P<0.05$ was assumed to be statistically significant. In the latter, excellent to moderate examinations are provided in weight-classes 1 - 3 . Reduced number in subgroups is due to poor datasets in weight-classes 4 and 5 .

\begin{tabular}{|c|c|c|c|c|c|c|c|c|c|c|c|c|}
\hline & \multicolumn{2}{|c|}{ Entirecohort } & \multicolumn{5}{|c|}{ Weight-class } & \multicolumn{3}{|c|}{ LV-Function } & \multicolumn{2}{|c|}{ Renal Function } \\
\hline & Diag. & Non-Diag. & 1 & 2 & 3 & 4 & 5 & Normal & Mild & Impaired & Normal & Impaired \\
\hline \multicolumn{13}{|l|}{ Per slice } \\
\hline All slices $(n=4841)$ & $\begin{array}{c}4066 \\
(84 \%)\end{array}$ & $\begin{array}{c}775 \\
(16 \%)\end{array}$ & $\begin{array}{c}205 \\
(100 \%)\end{array}$ & $\begin{array}{l}1038 \\
(85 \%)\end{array}$ & $\begin{array}{l}1757 \\
(94 \%)\end{array}$ & $\begin{array}{c}678 \\
(78 \%)\end{array}$ & $\begin{array}{c}388 \\
(57 \%)\end{array}$ & $\begin{array}{l}2101 \\
(82 \%)\end{array}$ & $\begin{array}{c}556 \\
(84 \%)\end{array}$ & $\begin{array}{c}379 \\
(72 \%)\end{array}$ & $\begin{array}{l}2317 \\
(80 \%)\end{array}$ & $\begin{array}{l}1507 \\
(90 \%)\end{array}$ \\
\hline $\begin{array}{l}\text { Slices in homogeneous } \\
\text { examinations } \\
\qquad(n=4071)\end{array}$ & $\begin{array}{c}3505 \\
(86 \%)\end{array}$ & $\begin{array}{c}566 \\
(14 \%)\end{array}$ & $\begin{array}{c}205 \\
(100 \%)\end{array}$ & $\begin{array}{c}633 \\
(86 \%)\end{array}$ & $\begin{array}{l}1694 \\
(94 \%)\end{array}$ & $\begin{array}{c}647 \\
(81 \%)\end{array}$ & $\begin{array}{c}326 \\
(62 \%)\end{array}$ & $\begin{array}{l}1807 \\
(83 \%)\end{array}$ & $\begin{array}{c}509 \\
(86 \%)\end{array}$ & $\begin{array}{c}315 \\
(82 \%)\end{array}$ & $\begin{array}{l}1978 \\
(81 \%)\end{array}$ & $\begin{array}{l}1285 \\
(92 \%)\end{array}$ \\
\hline \multicolumn{13}{|l|}{ Per patient } \\
\hline $\begin{array}{l}\text { All examinations } \\
\qquad(n=84)\end{array}$ & $\begin{array}{c}49 \\
(58 \%)\end{array}$ & $\begin{array}{c}35 \\
(42 \%)\end{array}$ & $\begin{array}{c}4 \\
(100 \%)\end{array}$ & $\begin{array}{c}12 \\
(57 \%)\end{array}$ & $\begin{array}{c}24 \\
(73 \%)\end{array}$ & $\begin{array}{c}8 \\
(57 \%)\end{array}$ & $\begin{array}{c}1 \\
(2 \%)\end{array}$ & $\begin{array}{c}28 \\
(61 \%)\end{array}$ & $\begin{array}{c}7 \\
(58 \%)\end{array}$ & $\begin{array}{c}3 \\
(43 \%)\end{array}$ & $\begin{array}{c}30 \\
(56 \%)\end{array}$ & $\begin{array}{c}16 \\
(61 \%)\end{array}$ \\
\hline $\begin{array}{c}\text { Homogeneous } \\
\text { examinations }(n=72)\end{array}$ & $\begin{array}{c}56 \\
(78 \%)\end{array}$ & $\begin{array}{c}16 \\
(22 \%)\end{array}$ & $\begin{array}{c}4 \\
(100 \%)\end{array}$ & $\begin{array}{c}10 \\
(77 \%)\end{array}$ & $\begin{array}{c}24 \\
(75 \%)\end{array}$ & $\begin{array}{c}8 \\
(62 \%)\end{array}$ & $\begin{array}{c}1 \\
(2 \%)\end{array}$ & $\begin{array}{c}27 \\
(68 \%)\end{array}$ & $\begin{array}{c}7 \\
(63 \%)\end{array}$ & $\begin{array}{c}3 \\
(50 \%)\end{array}$ & $\begin{array}{c}30 \\
(65 \%)\end{array}$ & $\begin{array}{c}14 \\
(63 \%)\end{array}$ \\
\hline
\end{tabular}

CM Protocol in non-ECG synchronized CTA with high-pitch technique.

Approximately $20 \%$ - 30\% of the cohort suffered from cardiac and/or renal disease, which is an adequate subgroup for statistical evaluation. Regarding cardiovascular risk factors, arterial hypertension is evident in 2/3, while other factors like smoking etc. yield approx. $1 / 4$ of the collective, without statistically significant gender dependent differences.

Normal distribution of patients in weight-classes 1 - 5 with peak in class 3 is observable.

\subsection{Assessment of Contrast Media Volume and Dose Estimation}

Corresponding to the short data acquisition duration of 4.5 seconds in mean, few contrast media had to be delivered (54 ml) for the entire aorta and iliacal vessels. Relatively much CM had to be applied for thoracic examinations according to $\mathrm{CM}$ protocol $(48 \mathrm{ml}$ ) in comparison to the entire vessel tree. Reason for this effect is, that just scan duration changes as a parameter between thoracic or thoracoabdominal examination for protocol purpose. The difference between these is 2.4 seconds, which has little impact on total CM volume. That means, with in mean $6 \mathrm{ml}$ more $\mathrm{CM}$ a thoracic examination is extendable to whole vessel tree.

Estimated effective dose could be lower in comparison to other studies [9], but this is in the first instance an effect of peak tube voltage fixed to $120 \mathrm{kV}$ due to department's standard settings.

\subsection{Assessment of Intravascular Contrast Enhancement}

Degree of evaluation is high containing $99.5 \%$ evaluable slices, because just a small number of exclusion on slice level (0.5\%, $n=29$ out of 4841$)$ had to be accepted.

By empirically defining diagnostic contrast enhancement with density greater or equal to 225 , which is similar compared to other studies [3], 84.2\% of all slices deliver diagnostic enhancement. Lowering diagnostic level from $250 \mathrm{HU}$ to $225 \mathrm{HU}$ seems adequate to recruit $15.4 \%$ of all slices scoring between 225 and $250 \mathrm{HU}$. We observed that image quality changes slightly by this.

Contrary to our protocol literature suggests a non-linear relationship between contrast enhancement and Iodine volume. Despite this inadequate contrast enhancement was observed in weight-class 5 on per slice, per vessel and per patient level even with linear increasement of iodine. This effect is visible in particular in downstream areas like the iliacal areas. That means CM dilutes too much by blood pool. Combination with impaired $\mathrm{LV}$ function increases this effect due to reduced CO and slowed blood flow.

While weight-class 1 and 3 yield excellent and moderate contrast enhancement, beside inefficacy in weightclass 5 a huge percentage of $43 \%$ non-diagnostic segments below $225 \mathrm{HU}$ appear in weight-class 2 and 4 . This phenomenon is not inline with decreasing vessel opacification from weight-class one to five. Reason for this 
seems to be imprecise bolus triggering. If bolus tracking fails, homogeneity of bolus is disturbed and a huge increase or decrease of density downstream is observable (Figure 1). By excluding two inhomogeneous examinations in weight-class 2, one each in weight-class 4 and 5 , number of diagnostic datasets rises relevantly.

Despite of imprecise triggering approximately $1 / 3$ of participants in each subgroup independently to cardial or renal disorders have non-diagnostic scored dataset, despite of $81 \%$ to $92 \%$ diagnostic slices in these subgroups. This effect is attributed to strict scoring rule of claiming $100 \%$ diagnostic slices per dataset and due to inadequate opacification in weight-classes 4 and 5.

It is striking, that in patients with mildly reduced LV-function mean density scores significantly higher than in patients with normal ejection fraction. This effect is observable in every segment and also on per patient level and bases upon an elevated and delayed peak vessel opacification caused by reduced cardiac output [3] [5]. A similar effect is visible in patients with impaired renal function, which cannot be explained by additionally reduced LV-function in this subgroup (fraction of patients with reduced LV Function in patients with or without renal disease: $30 \%$ vs. $34 \%$ ) determine.

\subsection{Limitations}

Main limitation is the inadequate vessel's contrast enhancement in patients bigger than $85 \mathrm{~kg}$ (weight-classes 4 and 5). Therefore use of protocol is not recommended; further studies with adjusted injection duration and eventually iodine flux are necessary. Another main limitation is the difficulty of precisely hitting bolus for data acquisition. Analysis reveals $14 \%(n=12)$ failures by demonstrating inhomogeneity of bolus. Reason for this is premature or delayed data acquisition, producing an ascending or descending bolus shape.

\section{Conclusion}

Analysed CM Protocol produces moderate but diagnostic vessel enhancement by using little CM (mean $=50.7 \pm$ $6 \mathrm{ml}$ ) in patients smaller than $86 \mathrm{~kg}$ (weight-class 1 to 3 ) independently to left ventricular or renal function. But, precise bolus triggering becomes crucial as shown by high failure rate of $14 \%$ in this study.

\section{Acknowledgements}

None.

\section{References}

[1] Fleischmann, D. (2005) How to Design Injection Protocols for Multiple Detector-Row CT Angiography (MDCTA). European Radiology, 15, E60-E65. http://dx.doi.org/10.1007/s10406-005-0166-X

[2] Weininger, M., Barraza, J.M., Kemper, C.A., Kalafut, J.F., Costello, P. and Schoepf, U.J. (2011) Cardiothoracic CT Angiography: Current Contrast Medium Delivery Strategies. AJR. American Journal of Roentgenology, 196, W260W272. http://dx.doi.org/10.2214/AJR.10.5814

[3] Bae, K.T. (2010) Intravenous Contrast Medium Administration and Scan Timing at CT: Considerations and Approaches. Radiology, 256, 32-61. http://dx.doi.org/10.1148/radiol.10090908

[4] Karlo, C., Leschka, S., Goetti, R.P., Feuchtner, G., Desbiolles, L., et al. (2011) High-Pitch Dual-Source CT Angiography of the Aortic Valve-Aortic Root Complex without ECG-Synchronization. European Radiology, 21, $205-212$. http://dx.doi.org/10.1007/s00330-010-1907-3

[5] Fleischmann, D. and Kamaya, A. (2009) Optimal Vascular and Parenchymal Contrast Enhancement: The Current State of the Art. Radiologic Clinics of North America, 47, 13-26. http://dx.doi.org/10.1016/j.rcl.2008.10.009

[6] Seifarth, H., Puesken, M., Kalafut, J.F., Wienbeck, S., Wessling, J., et al. (2009) Introduction of an Individually Optimized Protocol for the Injection of Contrast Medium for Coronary CT Angiography. European Radiology, 19, 2373-2382. http://dx.doi.org/10.1007/s00330-009-1421-7

[7] Yang, W.J., Chen, K.M., Liu, B., Pang, L.F., Zhang, H., et al. (2012) Contrast Media Volume Optimization in HighPitch Dual-Source CT Coronary Angiography: Feasibility Study. The International Journal of Cardiovascular Imaging, 29, 245-252.

[8] Goetti, R., Baumuller, S., Feuchtner, G., Stolzmann, P., Karlo, C., et al. (2010) High-Pitch Dual-Source CT Angiography of the Thoracic and Abdominal Aorta: Is Simultaneous Coronary Artery Assessment Possible? American Journal of Roentgenology, 194, 938-944. http://dx.doi.org/10.2214/AJR.09.3482

[9] Bolen, M.A., Popovic, Z.B., Tandon, N., Flamm, S.D., Schoenhagen, P. and Halliburton, S.S. (2012) Image Quality, 
Contrast Enhancement, and Radiation Dose of ECG-Triggered High-Pitch CT versus Non-ECG-Triggered StandardPitch CT of the Thoracoabdominal Aorta. American Journal of Roentgenology, 198, 931-938.

http://dx.doi.org/10.2214/AJR.11.6921

[10] Puippe, G.D., Winklehner, A., Hasenclever, P., Plass, A., Frauenfelder, T. and Baumueller, S. (2012) Thoraco-Abdominal High-Pitch Dual-Source CT Angiography: Experimental Evaluation of Injection Protocols with an Anatomical Human Vascular Phantom. European Journal of Radiology, 81, 2592-2596. http://dx.doi.org/10.1016/j.ejrad.2011.12.021

[11] Beeres, M., Schell, B., Mastragelopoulos, A., Herrmann, E., Kerl, J.M., et al. (2012) High-Pitch Dual-Source CT Angiography of the Whole Aorta without ECG Synchronisation: Initial Experience. European Radiology, 22, 129-137. http://dx.doi.org/10.1007/s00330-011-2257-5 